\title{
Hyperthermic intraperitoneal chemotherapy as consolidation treatment of advanced stage ovarian cancer
}

\author{
Jieun Ko, MD ${ }^{1}$, Hyeong In Ha, MD², Min Chul Choi, MD³, Sang Geun Jung, MD, PhD ${ }^{3}$, Hyun Park, MD, PhD ${ }^{3}$, \\ Won Duk Joo, MD, PhD', Seung Hun Song, MD, $\mathrm{PhD}^{3}$, Chan Lee, MD, $\mathrm{PhD}^{3}$, Joon Mo Lee, MD, PhD \\ 'Department of Obstetrics and Gynecology, CHA Bundang Medical Center, CHA University, Seongnam; 'ㄹepartment of Obstetrics and Gynecology,
Pusan National University Yangsan Hospital, Yangsan; ${ }^{3}$ Comprehensive Gynecologic Cancer Center, CHA Bundang Medical Center, CHA University,
Seongnam, Korea
}

Objective

To investigate the therapeutic efficacy of hyperthermic intraperitoneal chemotherapy (HIPEC) as consolidation treatment after completing first-line treatment in patients with advanced ovarian cancer.

\section{Methods}

A retrospective chart review was conducted on patients treated at the Comprehensive Gynecologic Cancer Center between January 2014 and 2019. Based on the inclusion criteria, 24 eligible patients who received HIPEC (paclitaxel $175 \mathrm{mg} / \mathrm{m}^{2}$, for 90 minutes, at $42^{\circ} \mathrm{C}$ ) (HIPEC group) as consolidation treatment after terminating the adjuvant chemotherapy were identified. Another 24 patients who met the inclusion criteria and did not receive HIPEC were matched, representing the non-HIPEC group. Disease-free survival (DFS) and overall survival (OS) were examined between the two groups.

\section{Results}

The median DFS was 28.7 and 24.2 months in the HIPEC and non-HIPEC groups, respectively $(P=0.688)$. The 3-year DFS rates in the HIPEC and non-HPEC groups were $39.5 \%$ and $32.6 \%$, respectively. However, the median OS was not determined. The 5 -year OS rates in the HIPEC and non-HIPEC groups were $86.2 \%$ and $81.3 \%$, respectively $(P=0.850)$. One patient developed grade 3 neutropenia. Other patients experienced mild adverse events after HIPEC.

\section{Conclusion}

This study suggests that consolidation HIPEC could not support the survival benefit after completing the first-line treatment for patients with advanced ovarian cancer, although no severe specific safety issues were found. Therefore, randomized trials evaluating consolidation HIPEC for the management of ovarian cancer are warranted.

Keywords: Ovarian neoplasm; Hyperthermic intraperitoneal chemotherapy; Intraperitoneal; Chemotherapy

\section{Introduction}

Ovarian cancer is considered to have the highest mortality rate among all gynecological malignancies. Due to the absence of specific symptoms in the early stage and reliable screening tests, the majority of patients are diagnosed at advanced stages, where cancer spreads beyond the ovaries to the peritoneal seeding [1]. The current standard treatment for advanced ovarian cancer involves maximum effort to reduce the tumor burden through optimal cytoreductive surgery (CRS), followed by platinum-based systemic chemo-
Received: 2021.03.12. Revised: 2021.05.31. Accepted: 2021.07.15. Corresponding author: Min Chul Choi, MD

Comprehensive Gynecologic Cancer Center, CHA Bundang Medical Center, 59 Yatap-ro, Bundang-gu, Seongnam 13496, Korea E-mail: oursk79@cha.ac.kr

https://orcid.org//0000-0003-4509-6731

Articles published in Obstet Gynecol Sci are open-access, distributed under the terms of the Creative Commons Attribution Non-Commercial License (http://creativecommons. org/licenses/by-nc/3.0/) which permits unrestricted non-commercial use, distribution, and reproduction in any medium, provided the original work is properly cited.

Copyright $\odot 2021$ Korean Society of Obstetrics and Gynecology 


\section{Obstetrics \& Gynecology Science}

Vol. 64, No. 5, 2021

therapy. If upfront CRS is not feasible, interval debulking surgery (IDS) can be performed after three cycles of neoadjuvant chemotherapy [2]. Despite these standard treatments, most patients experience relapse and eventually die [3]. The 5-year survival rate of women diagnosed with advanced ovarian cancer has changed slightly over the past few years but is still around $20-30 \%$. Therefore, several innovative attempts have been made to enhance the efficacy of conventional treatments and improve survival outcomes.

Intraperitoneal (IP) chemotherapy is one of the attempted methods. Tumors in advanced ovarian cancer primarily spread to the peritoneal cavity, the main site of tumor recurrence. Therefore, local treatment strategies appear to be an ideal approach. Intravenous (IV) chemotherapy combined with IP chemotherapy significantly improved progressionfree survival (24 vs. 18 months; $P=0.05$; risk ratio [RR], 0.80 ) and overall survival (OS; 66 vs. 50 months; $P=0.03 ; R R, 0.75$ ) compared with those of conventional IV chemotherapy in patients with optimally debulked stage III ovarian cancer [4]. IP chemotherapy increases drug concentration within the abdominal cavity multiple folds, and significantly delayed drug clearance from the peritoneal cavity prolongs the duration of drug exposure. However, some factors, including catheterrelated problems, toxicities, and postoperative adhesions that may hinder penetration, have been identified as potential barriers to applying this approach in routine clinical practice $[4,5]$.

Hyperthermic IP chemotherapy (HIPEC) is a modified and attenuated IP chemotherapy method that delivers chemotherapy into the peritoneal cavity during surgery under hyperthermic conditions. Delivering IP chemotherapy at the end of surgery and subsequent extraction can circumvent most drawbacks of IP chemotherapy while maintaining its advantages. Hyperthermia ranging from $42^{\circ} \mathrm{C}$ to $45^{\circ} \mathrm{C}$ has a cytotoxic effect on tumor cells, enhances the penetration of the chemotherapy, and induces tumor cell death via multiple mechanisms. These include impairment of DNA repair, inhibition of angiogenesis, and induction of apoptosis [6-8].

In 2018, van Driel et al. [9] reported a randomized phase III trial to evaluate the efficacy of HIPEC during IDS after neoadjuvant chemotherapy in patients with stage III ovarian cancer. A significant advantage in recurrence-free survival (14.2 vs. 10.7 months, $P=0.003$ ) and OS (45.7 vs. 33.9 months, $P=0.002$ ) was observed in the IDS with HIPEC group as compared to those in the IDS-alone group, without higher inci- dence of adverse events. Despite these encouraging results, the effectiveness of HIPEC as consolidation therapy remains controversial. Therefore, this study aimed to investigate the therapeutic efficacy of HIPEC as consolidation treatment after completing first-line treatment for patients with advanced ovarian cancer.

\section{Materials and methods}

A retrospective chart review was conducted on patients treated at the Comprehensive Gynecologic Cancer Center, Bundang CHA Medical Center, between January 2014 and 2019. A total of 49 patients undergoing HIPEC met the following inclusion criteria and were selected as study candidates: (1) pathological confirmation of stage III or higher epithelial ovarian, fallopian tube, or peritoneal cancer based on the International Federation of Gynecology and Obstetrics (FIGO) classification 2014 and (2) partial or complete response after the optimal primary CRS and 6-9 cycles of standard platinum-based adjuvant chemotherapy. Among them, 24 patients who received HIPEC as the consolidation treatment were classified into the HIPEC group, and 25 were excluded for the following reasons: HIPEC performed with primary CRS $(n=1)$, IDS $(n=11)$, or secondary CRS $(n=9)$, and pathologic confirmation of pseudomyxoma peritonei $(n=4)$. Another 24 patients who met the criteria and did not undergo HIPEC were matched to the non-HIPEC group. This study was approved by the institutional review board (CHA IRB 2018-11-049).

HIPEC was performed within 3 weeks after completion of adjuvant chemotherapy, based on the eligibility criteria. The patient was placed in the supine position in the operating room and administered general anesthesia. A midline incision was made and the peritoneal cavity was comprehensively explored. If any suspicious lesions were detected, they were surgically removed, and delicate adhesiolysis was performed. After confirming the absence of tumor remnants and adhesion formation, HIPEC was initiated using the open coliseum method. The procedure was performed by three gynecologic oncologists (J. M. L, C. L, and M. C. C.).

Paclitaxel was administered intraperitoneally at a dose of $175 \mathrm{mg} / \mathrm{m}^{2}$ and mixed in 2,500-3,000 mL of normal saline. The diluted solution was heated at a target temperature of $41.5-42.0^{\circ} \mathrm{C}$ and continuously circulated into the abdomi- 


\section{Obstetrics \& Gynecology Science}

Jieun Ko, et al. Consolidation HIPEC in ovarian cancer

nal cavity using a Belmont hyperthermic infusion pump (Belmont Instrument Corporation, Billerica, MA, USA). The temperature was measured using two IP probes. Two inflow tubes were placed in the pelvis and upper abdominal cavity at an infusion rate of $1,000 \mathrm{~mL} / \mathrm{min}$, whereas two other outflow tubes were placed in both paracolic gutters. The perfusion procedure was maintained for 60-90 minutes after 10 minutes of pre-heating. After completing the circulation procedure, chemotherapy-diluted fluid was extracted, and the cavity was irrigated with lactated Ringer's solution. After thorough hemostasis and identification of the impaired site, the incision site was closed layer-by-layer.

Patients who were confirmed with any residual pathologic results from biopsy or peritoneal cytology received 3-4 cycles of additional chemotherapy after recovery from HIPEC. Disease-free survival (DFS) was defined as the time interval from the completion of first-line treatment to the clinical, radiological, or serological recurrence of progression, whereas overall survival (OS) was defined as the time interval from the completion of the first-line treatment to the occurrence of all-cause death in the HIPEC and non-HIPEC groups. Data were censored at the date of the last contact for patients

Table 1. Clinico-pathologic characteristics of patients

\begin{tabular}{|c|c|c|c|}
\hline & HIPEC $(n=24)$ & Non-HIPEC $(n=24)$ & $P$-value \\
\hline Age (yrs) & $49.2(24-57)$ & $55.9(27-72)$ & 0.038 \\
\hline $\mathrm{BMI}\left(\mathrm{kg} / \mathrm{m}^{2}\right)$ & $24.0(19.6-32.5)$ & $24.8(18.4-37.1)$ & 0.948 \\
\hline FIGO Stage & & & 0.590 \\
\hline$\| \mathrm{A}$ & $2(8)$ & $4(17)$ & \\
\hline$\| \mathrm{B}$ & $4(17)$ & $1(4)$ & \\
\hline IIIC & $18(75)$ & $19(79)$ & \\
\hline Tumor grade & & & 0.645 \\
\hline Grade 1 & $3(13)$ & $1(4)$ & \\
\hline Grade 2/3 & $21(87)$ & $23(96)$ & \\
\hline Histology & & & 0.696 \\
\hline High grade serous & $18(75)$ & $18(75)$ & \\
\hline Low grade serous & $2(8)$ & $1(4)$ & \\
\hline Mucinous & $2(8)$ & $2(8)$ & \\
\hline Clear cell & $2(8)$ & $2(8)$ & \\
\hline Endometrioid & 0 & $1(4)$ & \\
\hline Germline $B R C A$ mutation status & & & 0.550 \\
\hline Mutated & $7(29)$ & $9(38)$ & \\
\hline Wild type & $17(71)$ & $15(62)$ & \\
\hline $\mathrm{PCl}$ at initial surgery & $13.8(6-23)$ & $12.7(5-23)$ & 0.518 \\
\hline CCR score ${ }^{a)}$ at initial surgery & & & 0.413 \\
\hline CC-O & $19(79)$ & $21(88)$ & \\
\hline CC-1 & $5(21)$ & $3(12)$ & \\
\hline Target agents after HIPEC & & & 0.450 \\
\hline Bevacizumab & 2 & 2 & \\
\hline Olaparib & 1 & 3 & \\
\hline Cycles of adjuvant chemotherapy & $6(6-9)$ & $6(5-9)$ & 0.296 \\
\hline
\end{tabular}

Values are presented as number (\%).

HIPEC, hyperthermic intraperitoneal chemotherapy; BMI, body mass index; FIGO, International Federation of Gynecology and Obstetrics; PCl, peritoneal cancer index; CCR, completeness of cytoreduction.

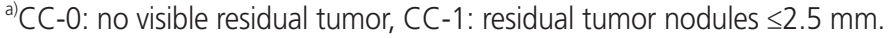




\title{
Obstetrics \& Gynecology Science
}

\author{
Vol. 64, No. 5, 2021
}

who remained alive and had no evidence of disease progression. The cutoff date for the data was set on April 30, 2021.

All statistical analyses were performed using IBM SPSS ver. 20.0 for Windows (IBM Corp., Armonk, NY, USA). Patient demographics analysis was conducted using the chi-squared test and analysis of variance. DFS and OS analyses were performed using the Kaplan-Meier method. Statistical significance was set at $P<0.05$.

\section{Results}

\section{Patient characteristics}

The clinicopathological characteristics are presented in Table 1. No statistical differences were observed in the body mass index, peritoneal cancer index score at the initial surgery, FIGO stage, tumor grade, histology, germline BRCA mutation status, and further treatment with target agents between the two groups. The median age of the non-HIPEC group was 6.7 years older than that of the HIPEC group (55.9 years vs. 49.2 years, $P=0.038$ ). As a result of the germline $B R C A$ test (specific test method was described in detail in a previous article [10]), mutations were found in approximately onethird of patients (29\% and $38 \%$ in the HIPEC and non-HIPEC groups, respectively). No difference was observed in patients who received bevacizumab or olaparib (one of the approved

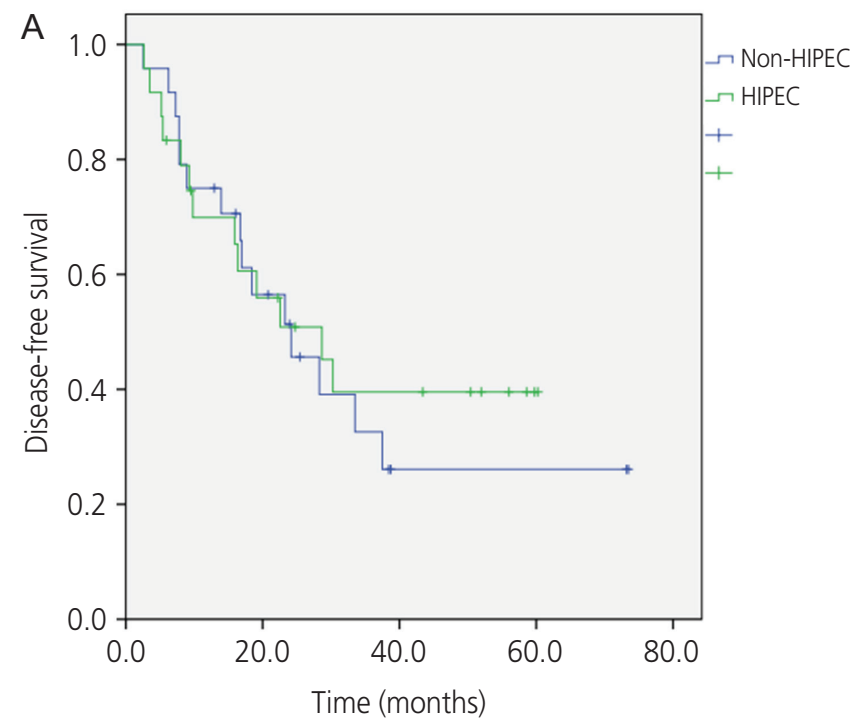

poly ADP-ribose polypmerase inhibitors in Korea) after HIPEC between the two groups.

In the HIPEC group, ten patients had positive results for residual tumors from biopsy or peritoneal cytology during HIPEC. They received 3-4 cycles of maintenance platinumbased chemotherapy after recovery from HIPEC.

\section{DFS and OS}

A total of $13(54.2 \%)$ and 15 (62.5\%) patients in the HIPEC and non-HIPEC groups, respectively, had experienced recurrence during the median follow-up of 47.6 months, with a median DFS of 28.7 (95\% confidence interval [Cl], 14.143.2) and $24.2(95 \% \mathrm{Cl}, 12.0-36.3)$ months, respectively $(P=0.688)$ (Fig. 1A), but without significant differences. The 3 -year DFS rates in the HIPEC and non-HPEC groups were $39.5 \%$ and $32.6 \%$, respectively. Four $(16.72 \%)$ and three patients $(12.5 \%)$ died in the HIPEC and non-HIPEC groups, respectively. However, the median OS was not reached. The 5 -year OS rates in the HIPEC and non-HIPEC groups were $86.2 \%$ and $81.3 \%$, respectively $(P=0.850)$ (Fig. 1 B).

\section{Adverse events}

Adverse events after HIPEC were assessed using the Common Terminology Criteria for Adverse Events (CTCAE) ver 5.0. The most common adverse events were fever and thrombocytopenia. Only one patient developed grade 3 neutropenia.

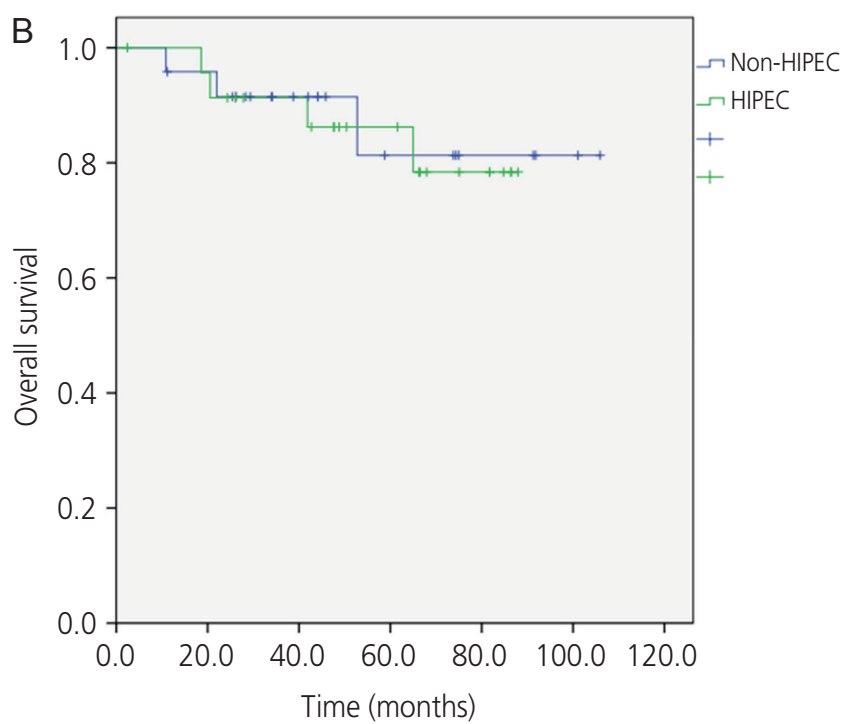

Fig. 1. Disease-free survival curve for the patients either treated with consolidation hyperthermic intraperitoneal chemotherapy (HIPEC) or cytoreductive surgery (CRS) and intravenous (IV) chemotherapy (A). Overall survival curve for the patlents either treated with consolidation HIPEC or CRS and IV chemotherapy (B). 


\title{
Obstetrics \& Gynecology Science
}

\author{
Jieun Ko, et al. Consolidation HIPEC in ovarian cancer
}

All patients recovered after conservative management.

\section{Discussion}

Previous studies have shown that intraperitoneal delivery of chemotherapy is associated with a several-fold increase in concentration in the abdominal cavity compared to that measured in the plasma after IV administration. Several randomized clinical trials have shown that IP combined with IV chemotherapy improves the survival rate in women with optimally debulked stage III ovarian cancer compared to that by conventional IV chemotherapy [11]. However, a recent phase III Gynecologic Oncology Group (GOG)-252 trial reported that IP chemotherapy is not beneficial for progression free survival (PFS) when compared to IV chemotherapy [12], although whether the addition of bevacizumab equalized outcomes remains unclear.

Since HIPEC is performed intraoperatively, the drug is administered uniformly under observation and abstracted thereafter. This can maximize the advantage of IP chemotherapy while reducing disadvantages such as pain and systemic toxicity. Although we observed one or more episodes of adverse events for each patient, all were grade one-two except for one episode of grade three neutropenia (absolute neutrophil count $[\mathrm{ANC}]<1,000 \mathrm{~mm}^{3}$ ). All patients tolerated and recovered after conservative care.

Various time points can be considered when performing HIPEC for the treatment of ovarian cancer, including upfront CRS, IDS, consolidation surgery, secondary CRS, and salvage CRS [13]. We considered it more effective and feasible to perform HIPEC as a consolidation treatment rather than performing primary debulking surgery because of tumor burden, operation time, and postoperative morbidity. In addition, lysis of postoperative adhesions before HIPEC application may increase the permeability of chemotherapy.

A randomized trial published by Spiliotis et al. [14] found a 13-month improvement in survival with the addition of HIPEC to secondary CRS, a benefit found in both platinumsensitive and platinum-resistant cohorts. More recently, van Driel et al.'s [9] randomized study on patients with stage III ovarian cancer undergoing neoadjuvant chemotherapy and IDS with or without HIPEC demonstrated similar results as previously described. Women who underwent IDS with HIPEC demonstrated a 3.5-and 12-month improvement in
PFS and OS, respectively.

Meanwhile, reports evaluating HIPEC for consolidation purposes have been limited. Bae et al. [15] retrospectively compared the survival rates of patients with ovarian cancer who underwent second-look surgery plus HIPEC with paclitaxel $(n=22)$ or carboplatin $(n=45)$ to no second-look surgery (conventional treatment, $\mathrm{n}=29$ ). In stage III disease, the 3 -year PFS rates were $56.3 \%$ and $16.7 \%$ in the HIPEC and control groups, respectively $(P=0.0028)$. In another retrospective matched-control study, Mendivil et al. [16] compared the survival rates of patients with advanced-stage ovarian cancer who were treated with consolidation HIPEC with carboplatin $(n=69)$ or not $(n=69)$. They showed more significant benefits of PFS in the HIPEC group (25.1 months) over that in the control group (20 months) $(P=0.024)$ although not shown in OS ( $P=0.29)$. A study conducted by Gori et al. [17] reported that patients treated with IP hyperthermic perfusion as consolidation therapy showed a median survival of 64.4 months, whereas the control group showed 60.1 months $(P=0.598)$. The mortality rate was lower in the intraperitoneal chemotherapy (IPCT)+hyperthermia (HT) group (44.8\% vs. $57.9 \%)$, but the differences were not statistically significant.

No statistically significant survival benefit was observed in the consolidation HIPEC group in this study. The median DFS was 28.7 vs. 24.2 months in the HIPEC and non-HIPEC groups, respectively $(P=0.688)$. Moreover, the 3-year DFS rates in the HIPEC and non-HPEC groups were $39.5 \%$ and $32.6 \%$, respectively. The 5 -year OS rates in the HIPEC and non-HIPEC groups were $86.2 \%$ and $81.3 \%$, respectively $(P=0.850)$ (Fig. 1). This may be due to limitations associated with retrospective studies with a small number of patients, and several differences in methodology and chemotherapy agents used in previous studies.

This study has some limitations. Several prognostic factors other than HIPEC may affect survival rate and should be considered. Age was an important prognostic factor. The patients in the HIPEC group were 6.7 years younger than those in the non-HIPEC group in our study (49.2 years vs. 55.9 years; Table 1). This could be a result of the reluctance to perform HIPEC in relatively elderly patients during the study period. This can also be a limitation of the retrospective comparative studies. Approximately 41.7\% (10/24) of patients in the HIPEC group received further maintenance chemotherapy for positive pathologic results after HIPEC, although a previous study suggested that three cycles of consolidation 


\section{Obstetrics \& Gynecology Science}

Vol. 64, No. 5, 2021

chemotherapy with paclitaxel and carboplatin might not prolong the survival rate in advanced ovarian cancer patients [18]. Although no statistical difference was observed, the use of target agents, such as bevacizumab [19] or PARP inhibitors [20], and BRCA status [21] (Table 1) may also have influenced the prognosis. Moreover, second-look surgery for consolidation is not currently accepted as a therapeutic strategy for advanced ovarian cancer. However, this surgery should be performed for the consolidation process of HIPEC, although it may be unnecessary.

According to the results of this study, no severe specific safety issues or survival benefits were found in HIPEC consolidation. Furthermore, there is a burden that patients must undergo unnecessary second-look surgery to perform HIPEC consolidation. Therefore, we could not routinely recommend HIPEC consolidation for patients with advanced-stage ovarian cancer, particularly outside of an approved clinical trial.

Several ongoing randomized trials have evaluated the benefits of HIPEC use in primary and recurrent ovarian cancer $[22,23]$. To date, most successes with HIPEC have been observed when used during IDS, and ongoing trials are examining the optimal timing of HIPEC application. These studies provide useful information about this therapeutic strategy.

\section{Conflict of interest}

No potential conflict of interest relevant to this article was reported.

\section{Ethical approval}

This study was approved by the institutional review board (CHA IRB 2018-11-049). The study was performed in accordance with the principles of the Declaration of Helsinki.

\section{Patient consent}

Written informed consent and the use of images from patients are not required for the publication.

\section{Funding information}

None.

\section{References}

1. Carlson KJ, Skates SJ, Singer DE. Screening for ovarian cancer. Ann Intern Med 1994;121:124-32.

2. du Bois A, Baert T, Vergote I. Role of neoadjuvant chemotherapy in advanced epithelial ovarian cancer. J Clin Oncol 2019;37:2398-405.

3. Cannistra SA. Cancer of the ovary. N Engl J Med 2004;351:2519-29.

4. Armstrong DK, Bundy B, Wenzel L, Huang HQ, Baergen $\mathrm{R}$, Lele $\mathrm{S}$, et al. Intraperitoneal cisplatin and paclitaxel in ovarian cancer. N Engl J Med 2006;354:34-43.

5. Jaaback K, Johnson N, Lawrie TA. Intraperitoneal chemotherapy for the initial management of primary epithelial ovarian cancer. Cochrane Database Syst Rev 2016;(1):CD005340.

6. Bakrin N, Classe JM, Pomel C, Gouy S, Chene G, Glehen O. Hyperthermic intraperitoneal chemotherapy (HIPEC) in ovarian cancer. J Visc Surg 2014;151:347-53.

7. Ohno S, Siddik ZH, Kido Y, Zwelling LA, Bull JM. Thermal enhancement of drug uptake and DNA adducts as a possible mechanism for the effect of sequencing hyperthermia on cisplatin-induced cytotoxicity in L1210 cells. Cancer Chemother Pharmacol 1994;34:302-6.

8. van de Vaart PJ, van der Vange N, Zoetmulder FA, van Goethem AR, van Tellingen O, ten Bokkel Huinink WW, et al. Intraperitoneal cisplatin with regional hyperthermia in advanced ovarian cancer: pharmacokinetics and cisplatin-DNA adduct formation in patients and ovarian cancer cell lines. Eur J Cancer 1998;34:148-54.

9. van Driel WJ, Koole SN, Sikorska K, Schagen van Leeuwen JH, Schreuder HWR, Hermans RHM, et al. Hyperthermic intraperitoneal chemotherapy in ovarian cancer. N Engl J Med 2018;378:230-40.

10. Choi MC, Heo JH, Jang JH, Jung SG, Park H, Joo WD, et al. Germline mutations of BRCA1 and BRCA2 in Korean ovarian cancer patients: finding founder mutations. Int J Gynecol Cancer 2015;25:1386-91.

11. Hess LM, Benham-Hutchins M, Herzog TJ, Hsu CH, Malone DC, Skrepnek GH, et al. A meta-analysis of 


\section{Obstetrics \& Gynecology Science}

Jieun Ko, et al. Consolidation HIPEC in ovarian cancer

the efficacy of intraperitoneal cisplatin for the frontline treatment of ovarian cancer. Int J Gynecol Cancer 2007;17:561-70.

12. Walker JL, Brady MF, Wenzel L, Fleming GF, Huang HQ, Disilvestro PA, et al. Randomized trial of intravenous versus intraperitoneal chemotherapy plus bevacizumab in advanced ovarian carcinoma: an NRG Oncology/Gynecologic Oncology Group Study. J Clin Oncol 2019;37:138090.

13. Mulier S, Claes JP, Dierieck V, Amiel JO, Pahaut JP, Marcelis $L$, et al. Survival benefit of adding Hyperthermic IntraPEritoneal Chemotherapy (HIPEC) at the different time-points of treatment of ovarian cancer: review of evidence. Curr Pharm Des 2012;18:3793-803.

14. Spiliotis J, Halkia E, Lianos E, Kalantzi N, Grivas A, Efstathiou $E$, et al. Cytoreductive surgery and HIPEC in recurrent epithelial ovarian cancer: a prospective randomized phase III study. Ann Surg Oncol 2015;22:1570-5.

15. Bae JH, Lee JM, Ryu KS, Lee YS, Park YG, Hur SY, et al. Treatment of ovarian cancer with paclitaxel- or carboplatin-based intraperitoneal hyperthermic chemotherapy during secondary surgery. Gynecol Oncol 2007;106:193200.

16. Mendivil AA, Rettenmaier MA, Abaid LN, Brown JV 3rd, Mori KM, Lopez KL, et al. Consolidation hyperthermic intraperitoneal chemotherapy for the treatment of advanced stage ovarian carcinoma: a 3 year experience. Cancer Chemother Pharmacol 2017;80:405-10.
17. Gori J, Castaño R, Toziano M, Häbich D, Staringer J, De Quirós DG, et al. Intraperitoneal hyperthermic chemotherapy in ovarian cancer. Int J Gynecol Cancer 2005;15:233-9.

18. Nayl B, Durando $X$, Pomel C, Dubray P, Mouret-Reynier $M$, Le Bouedec $G$, et al. Six versus nine cycles of paclitaxel-carboplatin as first-line chemotherapy in patients with newly diagnosed epithelial advanced ovarian cancer. J Clin Oncol 2009;27(15_suppl):e16535.

19. Tewari KS, Burger RA, Enserro D, Norquist BM, Swisher EM, Brady MF, et al. Final overall survival of a randomized trial of bevacizumab for primary treatment of ovarian cancer. J Clin Oncol 2019;37:2317-28.

20. Mirza MR, Coleman RL, González-Martín A, Moore KN, Colombo N, Ray-Coquard I, et al. The forefront of ovarian cancer therapy: update on PARP inhibitors. Ann Oncol 2020;31:1148-59.

21. Huang YW. Association of BRCA1/2 mutations with ovarian cancer prognosis: an updated meta-analysis. Medicine (Baltimore) 2018;97:e9380.

22. Cowan RA, O'Cearbhaill RE, Zivanovic O, Chi DS. Current status and future prospects of hyperthermic intraoperative intraperitoneal chemotherapy (HIPEC) clinical trials in ovarian cancer. Int J Hyperthermia 2017;33:54853.

23. Riggs MJ, Pandalai PK, Kim J, Dietrich CS. Hyperthermic intraperitoneal chemotherapy in ovarian cancer. Diagnostics (Basel) 2020;10:43. 